\title{
Evolutution of Sum Rules and Positivity Constraints
}

\section{O.V. Teryaev* JINR, 141980, Dubna, Russia}

E-mail: teryaevetheor.jinr.ru

\begin{abstract}
The preservation of sum rules andpositivity constraints is naturally provided by the kinetic descriptution of QCD evolution equations. It implies the irreversibility manifested in the existence of "scale arrows". The sign change in the course of TMD evolution at $Q \sim Q_{T}$ is interpreted as change of scale arrow direction. The Burkardt sum rule is related to momentum conservation and equivalence principle by a sort of analytic continuation. The claimed way of its evolution means its "spontaneous" conservation implied by the boundary condition, analogously to twist-3 contribution to Burkhardt-Cottingam sum rule. It is compatible with its validity separately for each flavour.
\end{abstract}

QCD Evolution 2016

May 30-June 03, 2016

National Institute for Subatomic Physics (Nikhef), Amsterdam

${ }^{*}$ Speaker. 


\section{Introduction}

The positivity constraints [1] and sum rules play the important role in the description of various parton distributions. Their stability against various types of QCD evolution is naturally explained in the framework of the kinetic interpretation of the latter leading to the phenomenon of irreversibility $[2,1]$ described by the appropriate "scale arrow". Here we address the issue of TMD evolution and suggest that it implies the "scale reversal" of the arrow at $Q \sim Q_{T}$ and substitution $W \rightarrow Y$ may be considered as its cure.

We also recall how Burkardt sum rule [4] may be related [3] to energy-momentum conservation by a sort of analytic continuation to complex domain of partonic momentum fractions. Its validity after account of QCD evolution [5] is due to the boundary condition and is similar to the stability of twist-3 contribution to Burkhardt-Cottingham sum rule [6]. Such mechanism allows for validity of Burkardt sum rule separately for each flavour.

\section{Kinetic evolution and scale arrows}

The DGLAP, BFKL and ERBL equations may be represented in the form of master equation

$$
\frac{d q(x, t)}{d t}=\int d y\left[w_{+}(y \rightarrow x) q(y, t)-w_{-}(x \rightarrow y) q(x, t)\right] .
$$

Here $q$ is a generic parton distribution and the role of "time" $t$ is played by the logs of either longitudinal momentum fraction for the Balitsky-Fadin-Kuraev-Lipatov (BFKL) equation or of the transverse momentum for the Dokshitzer-Gribov-Lipatov-Altarelli-Parisi (DGLAP) and Efremov-RadyushkinBrodsky-Lepage (ERBL)equations. Also, the gain $w_{+}$and loss $w_{-}$probabilities are, generally speaking different, like in BFKL case, where relation $w_{+}(t) \geq w_{-}(t)$ leads to the gluons fission.

The master form implies the irreversibility of equations, so that the positivity of distribution is preserved for growing $t$ and should be violated for backward evolution. The later can be also understood as an impossibility, in general, to represent boundary collision at some $t_{0}$ as a result of the evolution from smaller $t_{1}$.

The "scale arrow" selecting the proper direction of evolutiion is directed to UV for DGLAP and ERBL equations and to IR for BFKL equation, and this distinction may be understood as a manifestation of "angle arrow".

For the TMD evolution the asymptotic nullification of the moment of TMD pdf much discussed here [7] means the violation of positivity for large $Q_{T}$. One may understand that as a reversal of scale arrow at $Q_{T} \sim Q$ which is also supported by the sign change of the corresponding log. The substitution of $\mathrm{W}$ by $\mathrm{Y}$ term [7] may be interpreted as a restoration of the direction of scale arrow.

\section{Energy momentum conservation and Burkardt sum rule}

The contribution of gluonic pole to energy momentum should be nullified [3] due to the absence of the relevant structure $p^{\mu} \varepsilon^{\nu S p n}$ in the matrix element $\left\langle p\left|T^{\mu v}\right| p>\right.$. As a result

$$
\sum_{q, G} \int d x_{1} d x_{2} \frac{T\left(x_{1}, x_{2}\right)}{x_{1}-x_{2}}=0 .
$$


As it seems that the pole coming from EOM should be treated in p.v. sense, this is an identity due to the symmetry of $T\left(x_{1}, x_{2}\right)$ coming from the T-invariance. However, incorporating the imaginary prescription implied by contour gauge and electromagnetic gauge invariance [8], one get the analytic continuation:

$$
\sum_{q, G} \int d x_{1} d x_{2} \frac{T\left(x_{1}, x_{2}\right)}{x_{1}-x_{2}+i \varepsilon}=0,
$$

resulting in the non-trivial relation equivalent to Burkardt sum rule

$$
\sum_{q, G} \int d x T(x, x)=0 .
$$

Recent study of its evolution [5] resulted in multiplicative renormalization, so that the sum rule is satisfied ("spontaneously") provided it is valid at some scale. This is similar to the situation with the twist 3 contribution to Burkhardt-Cottingham sum rule [6]. It interesting that this sum rule is related to rotational invariance. The resuting angular momentum conservation, in turn, is combined with momentum conservation when the Ji's sum rules and equivalence principle is considered, being related to Burkardt sum rules [9].

Note that such way of preservation of Burkardt sum rule allows one to generalize it [3] to be valid for each flavour separately, which should lead to nodes [10] and may be related to the extension of equivalence principle [11].

\section{Conclusions}

The breaking of the positivity of TMD distributions may be related to the scale arrow reversal cured by substitution of $\mathrm{W}$ by Y. evolution of Burkardt sum rule may be similar to that BurkhardtCottingham sum rule implying its validity for separate flavours.

I am indebted to Daniel Boer, Piet Mulders and Alexei Prokudin for invitation to this interesting conference, warm hospitality in Amsterdam and interesting discussions.

\section{References}

[1] X. Artru, M. Elchikh, J. M. Richard, J. Soffer and O. V. Teryaev, Phys. Rept. 470 (2009) 1 doi:10.1016/j.physrep.2008.09.004 [arXiv:0802.0164 [hep-ph]].

[2] O. V. Teryaev, Phys. Part. Nucl. 36 (2005) S160.

[3] O. V. Teryaev, AIP Conf. Proc. 1654 (2015) 060007. doi:10.1063/1.4915990 O. Teryaev, EPJ Web Conf. 85 (2015) 02008. doi:10.1051/epjconf/20158502008

[4] M. Burkardt, Phys. Rev. D 69 (2004) 057501 doi:10.1103/PhysRevD.69.057501 [hep-ph/0311013].

[5] J. Zhou, Phys. Rev. D 92 (2015) no.7, 074016 doi:10.1103/PhysRevD.92.074016 [arXiv:1507.02819 [hep-ph]].

[6] A. Ali, V. M. Braun and G. Hiller, Phys. Lett. B 266 (1991) 117. doi:10.1016/0370-2693(91)90753-D

[7] J. Collins, L. Gamberg, A. Prokudin, T. C. Rogers, N. Sato and B. Wang, Phys. Rev. D 94 (2016) no.3, 034014 doi:10.1103/PhysRevD.94.034014 [arXiv:1605.00671 [hep-ph]]. 
[8] I. V. Anikin and O. V. Teryaev, Phys. Lett. B 690 (2010) 519 doi:10.1016/j.physletb.2010.05.072 [arXiv:1003.1482 [hep-ph]].

[9] O. V. Teryaev, AIP Conf. Proc. 915 (2007) 260 doi:10.1063/1.2750776 [hep-ph/0612205].

[10] D. Boer, Phys. Lett. B 702 (2011) 242 doi:10.1016/j.physletb.2011.07.006 [arXiv:1105.2543 [hep-ph]].

[11] O. V. Teryaev, Front. Phys. (Beijing) 11 (2016) no.5, 111207. doi:10.1007/s11467-016-0573-6; hep-ph/9904376. 